\title{
Bronchiectasis and rheumatoid arthritis: a clinical study
}

\author{
M J McMahon, D R Swinson, S Shettar, R Wolstenholme, C Chattopadhyay, P Smith, \\ P Johns, N H Crosby
}

\begin{abstract}
Objectives-To examine the relation between rheumatoid arthritis (RA) and bronchiectasis (BR).

Methods-Disease activity, outcome, extra-articular manifestations, and laboratory features were compared in 32 patients with BR and RA (RA-BR group), 32 matched patients with $R A$ without $B R$ (RA group), and 31 patients with BR but without arthritis (BR group).

Results-In 30 of the 32 (94\%) patients with RA-BR, BR preceded RA. There was no functional or radiological difference between the RA-BR and RA groups, and except for xerophthalmia, which was more common in patients with RA-BR than patients with RA, there was no difference in extra-articular or laboratory features.

Conclusions-Bronchiectasis does not lead to a more aggressive disease course in RA and, despite the recognised association, BR is not an extra-articular manifestation of rheumatoid disease.
\end{abstract}

(Ann Rheum Dis 1993; 52: 776-779)

An association between rheumatoid arthritis (RA) and bronchiectasis (BR) is well recognised, ${ }^{1-3}$ and similarities exist between these disorders. Both are associated with autoantibodies and abnormal serum immunoglobulin levels, and these may reflect disease severity in $\mathrm{BR}^{4}$ and RA. ${ }^{56}$ This paper reports a study of whether such abnormalities might be additive in the presence of the two diseases, whether this is reflected in more severe RA or $B R$, and discusses explanations for the association of these disorders in the light of the findings.

South Manchester,

Manchester, United

Kingdom

M J McMahon

Royal Albert Edward

Infirmary, Wigan,

United Kingdom

S Shettar

$\mathrm{R}$ Wolstenholme

Royal Preston

Hospital, Preston,

United Kingdom

P Johns

Correspondence to:

Dr M J McMahon,

Dumfries and Galloway

Royal Infirmary, Bankend

Road, Dumfries DG1 4AP,

United Kingdom.

Accepted for publication 22 July 1993

Patients and methods

Thirty two patients with RA and BR (RA-BR group) were identified from a computerised database of about 3000 patients attending a rheumatology outpatient clinic. Thirty two patients with RA alone (RA group) matched with these for age, sex, and duration of arthritis were then identified from the same source. A further 31 patients known to have BR but not RA were identified from a chest clinic in the same area (BR group). Only rheumatoid subjects with definite or classical RA by the American Rheumatism Association criteria ${ }^{7}$ were included. Patients with BR were identified initially on the basis of a known clinical diagnosis and all had chronic productive coughs, persistent coarse crackles on chest auscultation, and an abnormal chest radiograph. When further assessed using Walker's criteria, ${ }^{8} 28$ of the patients with RABR were classed as 'definite', and this was confirmed in 17 by previous bronchography or at operation. The remaining four subjects with RA-BR were 'probable' by clinical criteria. All 31 patients with $\mathrm{BR}$ were classified as 'definite', with confirmation in 22 from previous bronchographic findings. There was a history of surgical excision (lobectomy) in seven of the patients with RA-BR and eight of the patients with $B R$.

Disease outcome, disease activity, extraarticular features, and drug treatment were recorded for each subject with rheumatoid disease. Outcome was assessed by the Steinbrocker functional capacity, health assessment questionnaire (HAQ), number of orthopaedic procedures performed, and by scoring of hand and foot radiographs by a modified Larsen's method: each hand proximal interphalangeal, metacarpophalangeal, wrist, and lateral four metatarsophalangeal joints were scored $0-5$ by comparison with standard radiographs, ${ }^{9}$ and the total score derived by multiplying the wrist scores by five and adding all scores, the maximum possible score being 190. Disease activity was assessed by grip strength, visual analogue score for pain, haemoglobin, platelet count, erythrocyte sedimentation rate (ESR), and C reactive protein. Extra-articular features were identified by clinical examination and review of each patient's case notes. Schirmer's tear test was performed in all subjects, including the BR group. Present and previous disease modifying drug treatment and any adverse reactions to these drugs were noted.

Personal details (age of onset of RA and BR, duration of disease, smoking and occupational histories, and the presence of other autoimmune disorders) were recorded and al subjects in the study underwent a respiratory assessment with spirometry and peak expiratory flow measurement. Reversibility of airflow obstruction was assessed by adminisSubjects whose forced expiratory volume in $15 \%$ were said to have reversible airflow obstruction. tering two puffs of salbutamol $(200 \mu \mathrm{g})$. one second $\left(\mathrm{FEV}_{1}\right)$ improved by more than 
Table 1 Demographic features of study groups. Values are number of patients or mean $( \pm 95 \% C I)$ value

\begin{tabular}{lccc}
\hline & $\begin{array}{l}R A-B R \\
(n=32)\end{array}$ & $\begin{array}{l}R A \\
(n=32)\end{array}$ & $\begin{array}{l}B R \\
(n=31)\end{array}$ \\
\hline Male & 9 & 9 & 12 \\
Smokers & 14 & 18 & 16 \\
Age of onset of RA (years) & $46(5)$ & $46(5)$ & - \\
Age of onset of BR (years) & $19(5)$ & - & $16(4)$ \\
Duration of BR (years) & $38(2)$ & - & $38(2)$ \\
\hline
\end{tabular}

Abbreviations: $\mathrm{RA}=$ rheumatoid arthritis; $\mathrm{BR}=$ bronchiectasis.

Sputum production, dyspnoea, and radiographic disease were used to assess the severity of BR. Each was graded 1-3: daily sputum production $<20 \mathrm{ml}=1,20-30 \mathrm{ml}=2,>30$ $\mathrm{ml}=3$; dyspnoea mild or absent $=1$, moderate $=2$, severe $=3$, radiographic disease, two or fewer zones $=1$, three or four zones $=2$, honeycombing $=3$. The sum of these grades was used as a crude severity score.

Serum IgG, IgA, IgM, rheumatoid factor (latex and RAHA), IgG antinuclear antibodies, IgM antinuclear antibodies, autoantibodies (antimitochondrial, smooth muscle, parietal cell, thyroglobulin, thyroid microsomal, and extractable nuclear antigens), C3, C4, CH50, $\mathrm{C} 1$ esterase inhibitor (C1-INH), circulating immune complexes, precipitins (to aspergillus, candida and Farmer's lung), $\mathrm{C}$ reactive protein, $\alpha_{1}$ antitrypsin, haptoglobin, haemoglobin, white blood cell count, platelets and ESR, urea and electrolytes, liver transaminases, bilirubin, serum protein and globulin, and 24 hour urinary protein excretion were assessed in all subjects.

\section{STATISTICS}

Results are presented as proportions or means with 95\% confidence intervals. Where proportions are given, $\chi^{2}$ or Fisher's exact tests have been used to test significance. Results with continuous data and normal distribution were assessed by $t$ tests and others by the Wilcoxon or Mann-Whitney $U$ tests. Where appropriate (i.e. RA-BR $v \mathrm{RA}$ ) paired tests were used.

\section{Results}

The sex distribution, age at onset of disease, and smoking history of each group were similar (table 1). In the RA-BR group the diagnosis of $B R$ preceded $R A$ in all but two patients.

Functional assessments, radiological damage to joints (table 2), and disease activity measures were similar in the two RA groups. The proportion of patients who had undergone large joint arthroplasty was similar in the RABR (16\%) and BR (25\%) groups $\left(\chi^{2}=0.54\right.$

Table 2 Disease outcome. Values are mean ( $\pm 95 \%$ CI)

\begin{tabular}{lcc}
\hline & $R A-B R$ & $R A$ \\
\hline Steinbrocker FC & $1.8(0 \cdot 4)$ & $1 \cdot 6(0 \cdot 3)$ \\
HAQ & $1 \cdot 2(0 \cdot 28)$ & $1 \cdot 17(0 \cdot 28)$ \\
Radiographic score & $45.9(12 \cdot 8)$ & $46 \cdot 2(15 \cdot 2)$ \\
\hline
\end{tabular}

Abbreviations: RA=rheumatoid arthritis; $B R=$ bronchiectasis $\mathrm{FC}=$ functional capacity; $\mathrm{HAQ}=$ health assessment questionnaire.
Table 3 Number of subjects in each group with autoantibodies

\begin{tabular}{llll}
\hline & $R A-B R$ & $R A$ & $B R$ \\
\hline RF (latex) & 24 & 24 & 1 \\
RF (RAHA) & 25 & 26 & 3 \\
IgG ANA & 6 & 12 & 4 \\
IgM ANA & 9 & 15 & 5 \\
Mitochondrial antibodies & 0 & 1 & 0 \\
Smooth muscle antibodies & 1 & 1 & 4 \\
Parietal cell antibodies & 1 & 2 & 0 \\
Extractable nuclear antigen & 3 & 1 & 0 \\
Anti-TG & 1 & 3 & 0 \\
Anti-TM & 2 & 3 & 1 \\
\hline
\end{tabular}

Abbreviations: $\mathrm{RA}=$ rheumatoid arthritis; $\mathrm{BR}=$ bronchiectasis $\mathrm{RF}=$ rheumatoid factor

$\mathrm{p}=0.39$ ), though when all orthopaedic procedures were considered, fewer patients in the RA-BR group had been treated in this way (19 $v 47 \% ; \chi^{2}=4.54 ; \mathrm{p}=0.03$ ). This might reflect a bias against minor procedures in patients with significant chest disease. The number of disease modifying drugs used was similar in the two RA groups and the drugs were used in similar proportions. Steroids by mouth had been used in 16 patients in the RA$\mathrm{BR}$ group compared with five in the RA group $\left(\chi^{2}=5.89 ; p=0.015\right)$ and two in the BR group, but whether these were initially prescribed for respiratory disease or RA was uncertain.

No significant differences existed in the frequency of autoantibodies in the RA-BR and $\mathrm{RA}$ groups, though as might be expected autoantibodies were found less commonly in the group with BR alone (table 3).

Increased serum IgA levels were found in a substantial proportion of subjects from all groups and reduced IgM levels were found more commonly in the two bronchiectasis groups than in the group with $\mathrm{RA}$ alone (Fisher's exact probability: RA-BR, $p=0.052$; $\mathrm{BR}, \mathrm{p}=0.006)$. Mean immunoglobulin levels (table 4), however, were similar in all three groups. Levels of complement C3, C4, $\mathrm{C} 1-\mathrm{INH}$ and $\mathrm{CH} 50$ did not differ between the groups, but lower levels of circulating immune complexes and acute phase reactants were found in patients with BR than in those with RA-BR. This was statistically significant for haptoglobin $(\mathrm{t}=2.88 ; \mathrm{p}=0.005)$ and $\alpha_{1}$ antitrypsin $(t=2.48 ; \mathrm{p}=0.016)$, but mean values remained within the normal ranges in all groups.

Table 4 Immunoglobulin levels and percentage of subjects in each group with levels above (high) or below (low) the reference range. Values are mean $( \pm 95 \% C I)$

\begin{tabular}{llll}
\hline Antibody to & $R A-B R$ & \multicolumn{1}{l}{$R A$} & \multicolumn{1}{l}{$B R$} \\
\hline IgG (g/l) & $13 \cdot 15(1 \cdot 14)$ & $14 \cdot 05(1 \cdot 37)$ & $13 \cdot 8(1 \cdot 69)$ \\
IgA (g/l) & $4 \cdot 14(0 \cdot 74)$ & $3 \cdot 51(0 \cdot 50)$ & $3 \cdot 30(0 \cdot 67)$ \\
IgM (g/l) & $1 \cdot 70(0 \cdot 81)$ & $1 \cdot 54(0 \cdot 26)$ & $1 \cdot 26(0 \cdot 22)$ \\
IgG & & & \\
High (\%) & $9(10)$ & $22(14)$ & $10(11)$ \\
Low (\%) & 0 & 0 & $3(6)$ \\
IgA & & & \\
High (\%) & $44(17)$ & $38(17)$ & $35(17)$ \\
Low (\%) & 0 & 0 & $3(6)$ \\
IgM & & & \\
High (\%) & $6(8)$ & $19(14)$ & $10(11)$ \\
Low (\%) & $19(14)$ & $3(6)$ & $29(16)$ \\
Any abnormality (\%) & $66(16)$ & $63(17)$ & $65(17)$ \\
\hline
\end{tabular}

Abbreviations: $\mathrm{RA}=$ rheumatoid arthritis; $\mathrm{BR}=$ bronchiectasis Normal values: IgG, $7 \cdot 0-17.0 \mathrm{~g} / \mathrm{l} ;$ IgA $0.7-3.5 \mathrm{~g} / \mathrm{l} ; \mathrm{IgM}$ $0 \cdot 7-2 \cdot 10 \mathrm{~g} / \mathrm{l}$. 
Table 5 Schirmer's tear test (millimetres wetting in five minutes) and percentage of subjects with less than $5 \mathrm{~mm}$ wetting in either eye. Values are mean ( $\pm 95 \%$ CI)

\begin{tabular}{llll}
\hline & $R A-B R$ & $R A$ & $B R$ \\
\hline Left & $10 \cdot 1(3 \cdot 5)$ & $16 \cdot 8(4 \cdot 8)$ & $16 \cdot 1(4 \cdot 3)$ \\
Right & $10 \cdot 3(3 \cdot 8)$ & $18 \cdot 7(5 \cdot 1)$ & $17 \cdot 3(4 \cdot 4)$ \\
Either eye $<5 \mathrm{~mm}(\%)$ & $56(17)$ & $22(14)$ & $32(16)$ \\
\hline
\end{tabular}

Abbreviations: $\mathrm{RA}=$ rheumatoid arthritis; $\mathrm{BR}=$ bronchiectasis.

Table 6 Spirometry (as absolute values and percentage of predicted values) with the percentage of subjects in whom airways obstruction reverses with bronchodilators. Values are mean $( \pm 95 \% C I)$

\begin{tabular}{lccc}
\hline & $R A-B R$ & $R A$ & $B R$ \\
\hline FEV $_{1}$ & $0 \cdot 89(0 \cdot 18)$ & $1 \cdot 98(0 \cdot 28)$ & $1 \cdot 19(0 \cdot 18)$ \\
FVC & $1 \cdot 75(0 \cdot 27)$ & $2 \cdot 98(0 \cdot 35)$ & $2 \cdot 01(0 \cdot 23)$ \\
Peak flow & $187(32)$ & $367(45)$ & $257(36)$ \\
Reversibility (\%) & $6(8)$ & $32(16)$ & $27(15)$ \\
Percentage of & & & \\
predicted value & & & \\
$\quad$ FEV & & & \\
FVC & $56(6)$ & $76(8)$ & $44(7)$ \\
\hline
\end{tabular}

Abbreviations: $\mathrm{RA}=$ rheumatoid arthritis; $\mathrm{BR}=$ bronchiectasis; $\mathrm{FEV}_{1}=$ forced expiratory volume in one second; $\mathrm{FVC}=$ forced vital capacity.

The incidence of extra-articular features of RA (nailfold infarcts, nodules, major vasculitis, Raynaud's phenomenon, pulmonary fibrosis) was similar in the two groups except for the more frequent finding of xerophthalmia in patients with RA-BR $\left(\chi^{2}=6.56 ; \mathrm{p}=0.010\right)$ (table 5)

Spirometric abnormalities were common in all groups, with $\mathrm{FEV}_{1}$ /forced vital capacity (FVC) ratios less than $75 \%$ in 31 patients with RA-BR, 26 with RA, and 27 with BR. As can be seen in table 6 , these were most marked in the groups with BR, and especially so in the RA-BR group, in whom there were also less reversibility with bronchodilators (Fisher's exact test, $p=0.036$ ). Bronchiectasis as assessed by sputum production, dyspnoea, and radiographs, however, appeared to be less severe in the RA-BR group (RA-BR $5.9(0.6)$; BR $6.8 \quad(0.6)$; Mann-Whitney U test, $\mathrm{p}=0.037)$

\section{Discussion}

Though we have not formally assessed our patients with BR by bronchography or computed tomography scanning, when assessed by Walker's criteria, 59 patients had definite $B R$ and four probable BR. We have therefore chosen to use the term BR, in keeping with other workers, ${ }^{3}$ rather than the term chronic bronchial suppuration as used by Bamji and Cooke. ${ }^{2}$

In this study the presence of BR did not adversely affect activity or outcome measures of arthritis in rheumatoid disease. Bronchiectasis does not therefore result in a more aggressive disease course in RA. Moreover, despite the association, there is no indication that BR is a systemic feature of rheumatoid disease. If it was, we would expect more laboratory abnormalities and certainly more systemic rheumatoid features in those patients with $B R$ and RA, but these were not found.
The proportion of patients with increased serum IgA, for example, was similar in all three patient groups, and of extra-articular features only xerophthalmia, as assessed by Schirmer's tear test, was more pronounced in the group with RA and BR.

The spirometric findings suggest that bronchiectatic lung in rheumatoid disease may differ slightly from bronchiectatic lung without rheumatoid disease, with more pronounced obstructive airways disease and less reversibility with bronchodilators. This conclusion, is, admittedly, open to criticism, given the history of lobectomy in several of the patients, which would alter their spirometry and make comparison with predicted values unreliable. The number of patients who had had an operation was, however, similar in the two groups, as was the duration of $B R$, so the findings may represent a true qualitative difference in bronchiectatic lung with and without rheumatoid disease. This difference could, however, merely reflect the effect of Sjögren's syndrome. This is said to be a factor in the development of obstructive airways disease in $\mathrm{RA}^{10}$ and may explain why the more severe obstructive defects were found in patients with RA-BR, the group with most xerophthalmia.

Several explanations have been proposed to account for the association between RA and BR. Bamji and Cooke suggest that RA or its treatment might lead to an increased incidence of respiratory tract infections ${ }^{2}$ and so predispose to BR. This explanation is difficult to accept in our study group as BR tended to precede RA. Bronchiectasis itself therefore might be a more likely source of the association. In established BR, for example, the antigenic stimuli of chronic suppuration could have a role in causing RA. ${ }^{8}$ This is supported by the finding that RA starts at a younger age in patients with BR. ${ }^{11}$ As $\mathrm{BR}$ is not associated with more severe rheumatoid disease, however, it is unlikely that chronic suppuration per se simply drives the rheumatoid disease. If lung infection is in some way causally related to the onset of RA it is probably by allowing exposure to a range of bacterial antigenic stimuli, some of which may trigger the disease in a genetically susceptible subject.

Another possibility is that the two diseases may share a common vulnerability. Studies of class II MHC antigen associations in patients with $\mathrm{RA}$ and $\mathrm{BR}^{12}$ have found weak associations with $\mathrm{DQB} 1 * 0201$ and DQA $1 *$ 0501, but this accounts for only a small number of the affected patients, and rheumatoid disease and $\mathrm{BR}$ occurring in isolation do not share associations. Our finding of xerophthalmia, a recognised extra-articular feature of $R A,{ }^{13}$ in significant numbers of patients with only $B R$ might suggest a further relation between these two disorders. If xerophthalmia was one facet of a more widespread exocrine dysfunction including reduced bronchial secretions (possibly preceding overt rheumatoid disease by several years), a predisposition to the development of 
$\mathrm{BR}$, by allowing more frequent respiratory infection, and RA, by the same mechanism in genetically susceptible subjects, could be postulated. If this is true, we might expect to find an increased incidence of BR in patients with primary Sjögren's syndrome, but this is not always seen. ${ }^{14} 15$ This could be explained by methodological differences such as BR not being specifically sought in these studies, or population differences in that disease duration tended to be shorter than in our study. More exciting, perhaps, is the fact that these studies have been in primary Sjögren's syndrome. Could it be the interplay between the disease processes of Sjögren's syndrome and rheumatoid disease that is important in the development of BR?

The association between RA and BR is now well recognised, and further investigation of this relation may reveal useful clues to the aetiopathogenesis of these disorders. In the short term, however, the patients affected can be reassured that BR will not adversely affect their RA.

1 Aronoff A, Bywaters E G L, Fearnley G R. Lung lesions in rheumatoid arthritis. $B M \mathcal{F}$ 1955; ii: 228-32.

2 Bamji A, Cooke N. Rheumatoid arthritis and chronic bronchial suppuration. Scand $\mathcal{f}$ Rheumatol 1985; 14: 15-21.
3 Solanki T, Neville E. Bronchiectasis and rheumatoid disease: is there an association? Br $\mathcal{F}$ Rheumatol 1992; 31:
: disease:

4 Hilton A M, Doyle L. Immunological abnormalities in bronchiectasis with chronic bronchial suppuration. British fournal of Diseases of the Chest 1978; 72: 207-16.

5 Sjoblom K G, Saxne T, Pettersson H, Wollheim F A. Factors related to the progression of joint destruction in
rheumatoid arthritis. Scand $\mathcal{R}$ Rheumatol 1984;13: 21-7.

6 Wollheim F A, Pettersson H, Saxne T, Sjoblom K G. Radiographic assessment in relation to clinical and biochemical variables in rheumatoid arthritis. Scand $f$ Rheumatol 1988; 17: 445-53.

7 Ropes M W, Bennett G A, Cobb S, Jacox R, Jessar R A. 1958 revision of diagnostic criteria for rheumatoid arthritis. Bull Rheum Dis 1958; 9: 175-6.

8 Walker W C. Pulmonary infections and rheumatoid arthritis. $Q \mathcal{F}$ Med 1967; 142: 239-51.

9 Larsen A, Dale K, Eek M. Standard radiographs of rheumatoid arthritis. Adopted by the Eular Standing Committee of International Clinical Studies Including Therapeutic Trials. Oslo: Department of Radiology, Oslo Sanitetsforening Rheumatism Hospital. Published courtesy of Eli Lilly Co, 1975.

10 Radoux V, Menard H A, Begin R, Decry F, Koopman W J. Airways disease in rheumatoid arthritis patients. One element of a general exocrine dysfunction. Arthritis Rheum 1987; 3: 249-56.

11 McMahon M J, Swinson D R. Younger onset of rheumatoid arthritis in patients with bronchiectasis. $\mathrm{Br} \mathcal{F}$ Rheumatol 1993; 32 (suppl 1): 125.

12 Hillarby M C, McMahon M J, Grennan D M, et al. HLA associations with pulmonary complications of rheumatoid disease. Br $\mathrm{F}$ Rheumatol. In press.

13 Hazleman B L, Watson P G. Ocular complications of rheumatoid arthritis. Clin Rheum Dis 1977; 3: 501-26.

14 Kelly C, Gardiner P, Pal B, Griffiths I. Lung function in Primary Sjögren's syndrome: a cross sectional and Primary Sjögren's syndrome: a cross secti
longitudinal study. Thorax 1991; 46: 180-3.

15 Constantopoulos S H, Papadimitriou C S, Moutsopoulos H M. Respiratory manifestations in primary Sjögren's syndrome. A clinical, functional and histologic study. Chest 1985; 88: 226-9. 